was the cause of the neuralgia it was difficult to say. The spine was not examined.

7. Mr. Harrinson. The heart of a child aged two years and two months. It was observed to be cyanosed within a short period from its birth. Its breathing was always short and its heart palpitating. It remained weak, never able to walk, and always cold. The blueness of the skin was increased by any excitement, as crying, \&c. It died from the supervention of pneumonia. After death both lungs were found nearly universally carnified. The right side of the heart was enormously distended with clots and fibrine; auricle and ventricle greatly enlarged. The auriculo-ventricular orifice was so much enlarged or dilated as to annihilate the functions of the valves. The pulmonary artery was greatly dilated; the ductus arteriosus impervious. On the left side : the auricle was very small; the foramen ovale open, its edges rounded; no valvular apparatus; the auricula absent. No appearance of the entrance, after a very careful examination, of any pulmonary, veins. Ventricle very small. The circulation must have been very peculiar, and tracing it from the right auricle, it would appear that a portion of blood would escape into the left auricle, ventricle, aorta, and general circulation in the usual way; but that the greater portion would pass into the right ventricle, the pulmonary artery, and lungs, as usual; that it would then be returned by the pulmonary veins into either the vena azygos, or into the superior cava, more probably the latter, and then into the right auricle. There would thus be in the right auricle a mixture of arterial and venous blood, and this mixture it would be which, passing to the left side, alone ministered to the wants of the body, in this manner accounting for its peculiar appearance and its imperfect development. The circulation would be carried on nearly independently of the left side of the heart, approaching the dicælious heart, as described by Hunter.

It was considered by the Society a deviation exceedingly rare. It is much to be regretted, that the termination of the pulmonary veins was not demonstrated. The heart was taken away for future examination, and the anomaly not discovered till too late.

\section{DISEASES OF THE LUNGS.}

8. Mr. Walford. A portion of inflamed lung, taken from a child aged 17 days, who two days before death had been seized with convulsions, which recurred three or four times.

9. Mr. Walford. A specimen of hepatized lung taken from a child dying from acute pneumonia.

(To be concluded in our next.)
PRACTICAL OBSERVATIONS ON NECROSIS

\section{IN THE LONG BONES.}

\author{
By W. S. Ore, M.D., Southampton.
}

It is my intention on the present occasion to say a very few words on the nature and treatment of necrosis, when it takes place in the internal part of the shaft of the long bones.

Although that celebrated surgeon, the late $\mathrm{Mr}$. William Hey, of Leeds, has handed down to us in his "Practical Observations in Surgery," some valuable remarks and directions on this subject, it appears to me, from his observations having been either overlooked or disregarded, that this disease does not meet, from the majority of surgeons, with the attention it deserves, nor the treatment it requires; and, consequently, that very many of these cases are allowed to go on month after month, year after year, without any real effort being made to remedy them, till at length, from the long continuance and increase of the disease, amputation is often resorted to as the only means left of curing, or rather getting rid of it, whilst, at the onset of the case, an easy and safe operation might have been adopted that would have prevented so serious a result.

When, from whatever cause, a portion of the internal structure of a bone becomes disorganised, and loses its. vitality, a curative process is at once set up by the surrounding sound parts, deep-seated, severe, and continued pain is felt in the bone affected, depriving the sufferer of his rest, and making sad havoc with his general health.

The surgeon examines the limb again and again, searching for some deep-seated abscess, but he finds none. Leeches, fomentations, and cataplasms are applied without effecting any mitigation of the pain; and the only remedy which affords some degree of relief, are repeated doses of laudanum.

After a while the periosteum is thickened, and the shaft of the bone becomes sore under pressure, and enlarged by the deposit of new bony matter around the dead or disorganised part. At length a small abscess is felt under the integuments, which makes its way through them, and gives relief to the patient.

Upon a careful examination the abscess is found to communicate through an opening in the new bone, which is like the perforation of a gimlet, with the cavity in which the sequestrum lies. If the blunt end of a probe be bent at an obtuse angle, and introduced through the perforation, we shall be enabled to ascertain the directions which the cavity takes; and by turning its blunt extremity upwards, supposing the limb to lie horizontally, we shall at once bring it into contact with the bony roof of the cavity, which is, in fact, the internal surface of the new bone.

We are thus made acquainted with the true state of the case, viz., that disorganization had taken place in a portion of the internal structure of the bone; that suppuration had necessarily followed; and that the new bone, deposited around the dead portion, had been perforated in order first to evacuate the matter, and secondly, the opening having been gradually enlarged, to allow of the sequestrum being thrown off.

This is precisely what takes place in the soft parts, when there is loss of vitality in some portion of the subcutaneous cellular tissue. Here we have severe 
pain, throbbing, redness, and thickening of the integuments, and suppuration beneath them, when, as it were, all at once a small opening takes place through the integuments, which discharges a fœtid matter, and gradually along with it, the sloughs of the tissue.

Necrosis is more frequently met with in children and young persons; and the tibia is most commonl the seat of the disease. The other cylindrical bones are also sometimes similarly involved.

In the treatment of these cases, the indication clearly is to enlarge the aperture, in order to remove the sequestrum. The sooner this is done the better. We shall be justified in doing it even before any natural opening has been formed, provided we are confident as to the nature and situation of the disease, upon the same principle that we are called upon to cut through aponeurotic texture, for the outlet of pus confined beneath it; but if we are not sufficiently certain of our diagnosis, (and this will generally be our position,) we are to wait till the bone has been naturally perforatedno longer, because, if we permit the disease to go on without enlarging the opening, the bone, from being constantly exposed to the matter accumulated in the cavity, will probably become more extensively disorganised, and the result will be, that instead of being able to remove the disease by one perforation of the trephine, we shall be under the necessity of making several more.

As soon, then, as a cavity in the internal structure of a bone is discovered, for example of the tibia, the limb being laid upon a pillow and firmly steadied at the knee and ankle, a conical incision is to be made through the orifice of the abscess down to the bone, and sufficiently long to allow the integuments to be dissected up, and the bone laid bare as far as the disease extends, which can generally be determined by the morbid condition of the periosteum, and by our exploration of the cavity below. If there be more than one aperture in the bone, the dissection is to be continued till the whole of them be fairly exposed.

Should we find only one, and that leading to a transverse or very limited cavity, the removal of a single circle of bone might be sufficient, which is to be sawed out by a moderately-sized conical trephine, cutting at its side as well as at its extremity; but if the cavity be found to extend longitudinally down the shaft, or if there be several openings, then so many circles are to be sawed out as the extent of the disease shall require. The intervening bridges of bone may be removed by Hey's saw, or, what is better, they may be cut out by a small chissel, and the gentle stroke of a mallet.

The cavity being freely exposed, the sequestrum, if loose, is to be removed at once; if not, it must be left to be disposed of by the natural process.

If the cavity consist of carious bone, which will sometimes be the case, the diseased surface of the cavity should be cleared away by a sharp gouge, after the manner recommended by Mr. Hey.

We shall occasionally find that the cavity contains a sequestrum, extending to a considerable length, both above and below the aperture, which, therefore, cannot be extracted through it. Under these circumstances the sequestrum is to be cut across in the open space we have made, which will enable us to draw out first one end and then the other. This was done in the following case, successfully treated by myself some years ago.

A fine young man, 21 years of age, consulted me for a severe pain of the upper third of the right arm, which he attributed to rheumatism from cold. It was treated accordingly, but without any good effect, and it soon became evident that the disease was of a much more serious nature.

The pain continued; was more and more severe, and required frequent doses of the tincture of opium for its alleviation. The bone was soon observed to be enlarged and the muscles attenuated. After several weeks a small abscess was discovered on the external surface of the arm at about the insertion of the deltoid muscle, when the severity of the pain subsided.

Upon examination, the abscess was found to communicate with a cavity in the internal structure of the shaft of the bone; and as there was no prospect of a cure by the natural efforts, a conical incision was made through the orifice of the abscess down to the periosteum, and the soft parts sufficiently dissected up to allow of the action of the trephine. Three circles of a thick bony case were sawed out, and the opening completed by Hey's saw. This brought into view a sequestrum extending both above and below the opening, the extreme length of which could not be determined by a common probe, and which of course could not be extracted. It therefore became necessary to cut it across; and by so doing, I was enabled to extract the two ends, which, when joined together, were found to correspond in length with the entire shaft of the humerus.

Upor examining the cavity of the new bone, from which the sequestrum had been extracted, it was found to have a smooth surface. The integuments were now laid down and covered by a poultice. The wound was afterwayds treated with simple dressings, and lastly with strips of adhesive plaster.

Under this treatment the case progressed most favourably. The cavity gradually became consolidated by bony deposit; and the young man completely recovered : the humerus, however, remained flattened where it had been perforuted by the trephine.

During the performance of the operation an interesting circumstance occurred, which I think is worthy of being recorded.

In cutting down through the muscles, the musculocutaneous nerve was unavoidably divided, which occasioned the instantaneous dropping of the hand. This appeared at first to be an unfortunate result, as it was feared it might occasion the permanent loss of the hand, and deprive him of the power of writing, for he could use his pen tolerably well.

To make up for so serious a deprivation in some degree, as soon as the state of the right arm would admit of it, he was sent to a schoolmaster to be taught to write with his left hand, which in about four months he accomplished; but at the end of this time I was gratified to observe a returning power in the right hand. The power gradually increased; and in the course of a few weeks he completely regained its use, and thus was enabled to write with both hands.

This was an important fact in physiology, inasmuch as it showed that the trunk of a nerve, though it be divided, and its ends kept apart for a considerable 
time, might eventually unite, and regain its normal functions.

I have treated several other cases of necrosis-most of them in the tibia-in the manner above recommended, and always with success.

Southampton, July 11, 1844.

\section{CASE OF SPONTANEOUS RUPTURE OF THE UTERUS.}

By W. Arnold, M.D., Kingston, Jamaica.

The following remarks were made by Dr. Henriques, who was first called to the patient on the 20th of November, 1842 :-

Mrs. Brown, a black woman, about 35 years of age, plethoric, of leuco-phlegmatic temperament, remarkably fat, is the mother of several children; in giving birth to them she invariably suffered, and indeed was ill prior and subsequent to delivery.

At ten o'clock this morning, whilst at breakfast, she was suddenly seized with uterine pains, by no means severe, and these pains ceased as suddenly, but were succeeded by nausea, continued syncope, and uterine hremorrhage. She was at the full term of gestation.

She was entirely free from pain; on the arrival of Dr. Henriques she complained however of great weakness. Several clots of blood were taken from the vagina, but no active hæmorrhage existed at the time; the neck of the uterus was soft, and dilated only to admit the introduction of the little finger; the pulse full, but easily compressed. Imagining that internal hæmorrhage was going on, she was bled, and took an opiate. Two hours after, upon examination, she was found precisely in the same state; the fulness of the pulse somewhat diminished, and the disposition to syncope more prolonged. Large doses of the secale cornutum were given, but this powerful medicine produced no effect.

At nine, p.m., there were evident signs of sinking, without any appreciable cause. No pain, no hæmorrhage, no further dilatation.

It was digreed to have a consultation, and I received a note from Dr. Henriques requesting me to meet him.

The patient was lying in a quiescent state, free from pain; the pulse weak, about 100 ; she replied to questions, but nothing satisfactory was elicited.

We agreed in the propriety of effecting delivery by forcibly dilating the uterus, which did not permit of a free examination, although I brought away one or the clots of blood, which nearly filled the vagina. Internal hæmorrhage was unquestionable, although not active, delivery at this juncture was evidently impracticable. Dr. Henriques proposed to try the effect of belladonna, with the view to dilatation, and to await one or two hours. At twelve o'clock the uterus admitted of a free examination, and delivery by turning was effected; the placenta was extracted entire, and what was equally surprising to us both, not a single drop of blood was lost, if we except one or two clots taken away just before the birth.

She died at three in the morning, without a struggle.

Post-mortem examination about noon on the 21 st. A triangular incision was made in the hypogastric region; in this incision the abdominal parietes were comprised, and on turning downwards the angular flap, the uterus was brought into view. Here was presented to us a scene as unexpected as it was extraordinary; immense clots of blood surrounded the uterus, filling up the whole abdominal cavity, the removal of which enabled us to lift the uterus, and now it was that we discovered an extensive rupture on. the posterior surface.

The uterus was accordingly dissected out, and carefully examined, its texture appeared unusually soft; the mucous surface bore evident traces of inflammatory action at different points, and the external serous covering also presented erysipelatous discolouration.

REMARKS.

This case is novel and extraordinary :-first, beeause the rupture appeared to be the result of inflammatory action, which gave no pain except that described when the injury took place, which pain was considered the premonitory pain of approaching labour; secondly, because softening of the uterine tissue is very rare; thirdly, because the fotus and placenta maintained their positions instead of escaping into the abdominal cavity.

Kingston, Jamaica, May 7, 1844.

\section{CASE OF INVERSIO UTERI.}

By William Joseph Square, Esq., Surgeon to the South Devon and East Cornwall Hospital, to the Plymouth Eye Infirmary, \&c.

TO THE EDITOR OF THE PROVINCIAL MEDICAL AND Sir,

In accordance with a request, expressed by $\mathrm{Mr}$. Crosse in a late number of your Journal, I beg to forward to you a case of inversion of the uterus, which will probably be interesting to some of your readers.

On the 14th of February, 1840, I was summoned in haste to Mrs. D., a powerful woman, having a large pelvis, who was delivered on the $3 d$ of the month, of twin children. She had been attended by Mr. who stated that her labour, (her first one,) had been perfectly natural, and completed in an hour and a quarter after he first visited her, that the placentæ were expelled with very trifling manual assistance, just sufficient to disengage them from the vagina, and that their expulsion was followed by a very small quantity of blood, certainly not more than three or four ounces. This statement accorded in every particular, with that of the patient and her friends.

For eight days she remained in bed, and was quite well. On the evening of the eighth day she dressed, and sat up, and while in the sitting posture experienced a trifling uterine hæmorrhage. Two days afterwards, while sitting up, this was repeated, and to a larger amount.

To-night, she had lost about a quart of florid blood. On my arrival she was seated on a chair, feeble, and very pale. I caused her to be placed in bed, and covered her with the sheet only. I then made firm pressure on the abdomen with a pad and bandage, and ordered one scruple of secale cornutum, infused in boiling water, to be taken every two hours.

15th. The hæmorrhage has ceased. A sanguineoserous discharge continues. She presents the ordinary constitutional symptoms of a large loss of blood. 